TITLE:

\title{
Quantum-confinement effects on conduction band structure of rectangular cross-sectional GaAs nanowires
}

\author{
AUTHOR(S): \\ Tanaka, H.; Morioka, N.; Mori, S.; Suda, J.; Kimoto, \\ $\mathrm{T}$.
}

\section{CITATION:}

Tanaka, H.... [et al]. Quantum-confinement effects on conduction band structure of rectangular cross-sectional GaAs nanowires. Journal of Applied Physics 2014, 115(5): 053713.

ISSUE DATE:

2014-02-07

URL:

http://hdl.handle.net/2433/192255

\section{RIGHT:}

Copyright 2014 American Institute of Physics. This article may be downloaded for personal use only. Any other use requires prior permission of the author and the American Institute of Physics. 


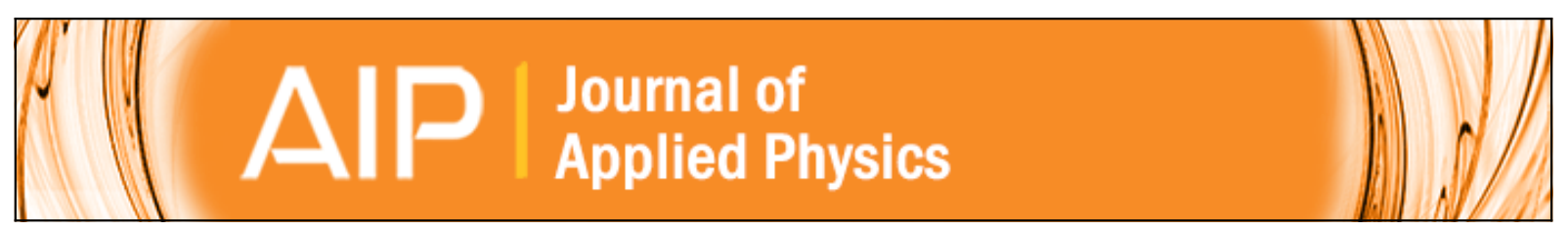

\section{Quantum-confinement effects on conduction band structure of rectangular cross- sectional GaAs nanowires}

H. Tanaka, N. Morioka, S. Mori, J. Suda, and T. Kimoto

Citation: Journal of Applied Physics 115, 053713 (2014); doi: 10.1063/1.4864490

View online: http://dx.doi.org/10.1063/1.4864490

View Table of Contents: http://scitation.aip.org/content/aip/journal/jap/115/5?ver=pdfcov

Published by the AIP Publishing

\section{Articles you may be interested in}

Conduction band structure in wurtzite GaAs nanowires: A resonant Raman scattering study

Appl. Phys. Lett. 100, 073102 (2012); 10.1063/1.3684837

Quantum-confinement effect on holes in silicon nanowires: Relationship between wave function and band structure

J. Appl. Phys. 109, 064318 (2011); 10.1063/1.3552593

Addendum: "Effective-mass enhancement and nonparabolicity in thin GaAs quantum wells" [J. Appl. Phys. 88, $6945(2000)]$

J. Appl. Phys. 91, 9435 (2002); 10.1063/1.1467969

Conduction band offset and electron effective mass in GalnNAs/GaAs quantum-well structures with low nitrogen concentration

Appl. Phys. Lett. 78, 2217 (2001); 10.1063/1.1362335

Effective-mass enhancement and nonparabolicity in thin GaAs quantum wells

J. Appl. Phys. 88, 6945 (2000); 10.1063/1.1321773

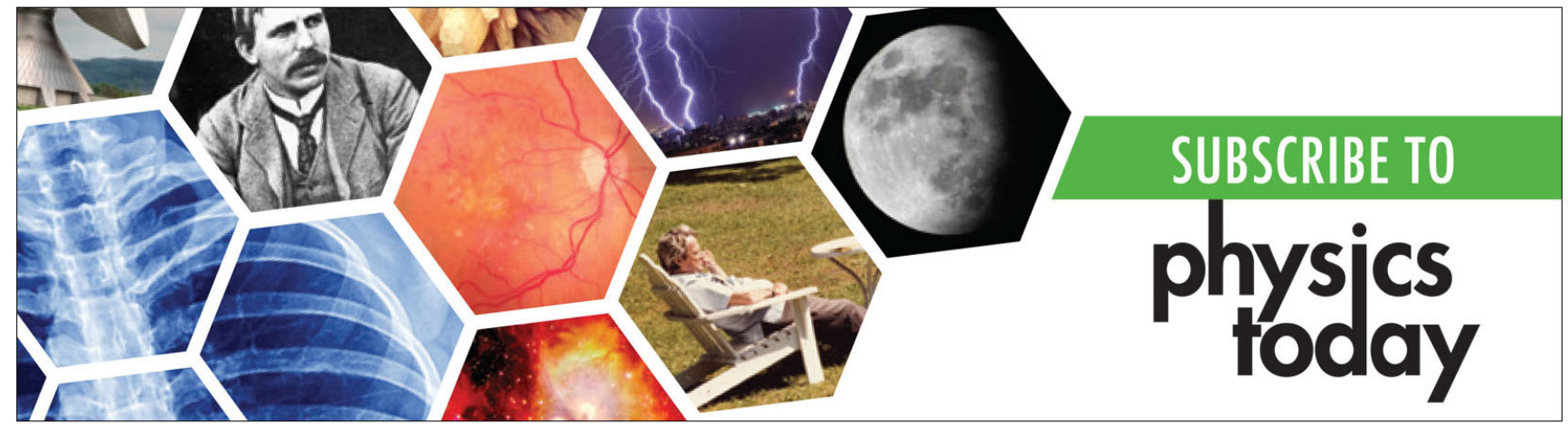




\title{
Quantum-confinement effects on conduction band structure of rectangular cross-sectional GaAs nanowires
}

\author{
H. Tanaka, ${ }^{\text {a) }}$ N. Morioka, S. Mori, J. Suda, and T. Kimoto \\ Department of Electronic Science and Engineering, Kyoto University, Kyoto 615-8510, Japan
}

(Received 20 November 2013; accepted 24 January 2014; published online 7 February 2014)

\begin{abstract}
The conduction band structure and electron effective mass of GaAs nanowires with various cross-sectional shapes and orientations were calculated by two methods, a tight-binding method and an effective mass equation taking the bulk full-band structure into account. The effective mass of nanowires increases as the cross-sectional size decreases, and this increase in effective mass depends on the orientations and substrate faces of nanowires. Among [001], [110], and [111]-oriented rectangular cross-sectional GaAs nanowires, [110]-oriented nanowires with wider width along the [001] direction showed the lightest effective mass. This dependence originates from the anisotropy of the $\Gamma$ valley of bulk GaAs. The relationship between effective mass and bulk band structure is discussed. @ 2014 AIP Publishing LLC. [http://dx.doi.org/10.1063/1.4864490]
\end{abstract}

\section{INTRODUCTION}

In integrated circuits, planer silicon metal-oxide-semiconductor field-effect transistors (MOSFETs) have been the core devices so far and their performance has been markedly improved by downscaling. However, the further scaling is facing difficulty mainly because of pronounced short channel effects. Since surrounding gate structure has excellent electrostatic control and can suppress short channel effects, ${ }^{1}$ nanowire MOSFETs have received increasing attention. ${ }^{2-4}$ On the other hand, III-V semiconductors with high electron mobility are promising as n-channel materials which can overcome the material limit of silicon MOSFETs, and studies on III-V MOSFETs are also attracting attention. ${ }^{5,6}$ For these reasons, studies on III-V semiconductor nanowire transistors have started in recent years. ${ }^{7,8}$ Since electron transport characteristics depend on the conduction band structure, it is important to understand the conduction band structure of III-V semiconductor nanowires for designing devices.

In this paper, we calculated the conduction band structure of rectangular cross-sectional nanowires made of GaAs, which is a typical III-V semiconductor. There have been several calculations on the conduction band structure of GaAs nanowires using various methods, an $s p^{3} s^{*}$ tight-binding (TB) method, ${ }^{9-11} s p^{3} d^{5} s^{*}$ TB method, ${ }^{12}$ empirical pseudo-potential method, ${ }^{13,14}$ first principles method. ${ }^{15-17}$ However, systematic research on the quantum confinement effects on the conduction band structure of rectangular cross-sectional GaAs nanowires with various wire orientations is still missing. In this work, we analyzed the dependencies of conduction band structure on the wire orientations and cross-sectional shapes. Then we gave a physical explanation to the calculated results based on the conduction band structure of bulk GaAs. Bulk GaAs has the conduction band minimum at $\Gamma$ valley, and it is often described by an isotropic effective mass. However, the $E-k$ dispersion of the $\Gamma$ valley has nonparabolicity and

${ }^{\text {a)} E l e c t r o n i c ~ m a i l: ~ t a n a k a @ s e m i c o n . k u e e . k y o t o-u . a c . j p . ~}$ anisotropy especially for large wavenumbers, which affect the electron effective mass in nanowires.

This paper is organized as follows. In Sec. II, the details of calculation methods and the structure of nanowires calculated in this study are explained. In Sec. III, several examples of the calculated conduction band structure are shown, and the width dependencies of quantum-confinement-induced energy shift and electron effective mass are presented. Section IV discusses the physical explanation of the dependence of effective mass on wire geometry. In Sec. V, we present favorable wire geometry for electron transport and conclude this paper.

\section{CALCULATION DETAILS}

\section{A. Calculation methods}

For the calculation of the conduction band structure of GaAs nanowires, we employed two different methods, a nearest-neighbor $s p^{3} d^{5} s^{*}$ TB method ${ }^{18,19}$ and a discrete effective mass equation (DEME) with the full-band $E-k$ dispersion of bulk GaAs. The calculation was performed by the custommade program. ${ }^{20}$ By comparing the results of TB and DEME, quantum confinement effects on electrons were analyzed.

In the $s p^{3} d^{5} s^{*}$ TB method, one $s$ orbital, three $p$ orbitals $\left\{p_{x}, p_{y}, p_{z}\right\}$, five $d$ orbitals $\left\{d_{y z}, d_{z x}, d_{x y}, d_{3 z^{2}-r^{2}}, d_{x^{2}-y^{2}}\right\}$, and one excited $s^{*}$ orbital are taken into account. Then the interactions between nearest neighbor atoms are considered to calculate energy band structure. Spin-orbit coupling can influence the effective mass in the conduction band. However, we confirmed that the effective mass calculated with and without spin-orbit coupling was almost the same. Thus, we neglected the spin-orbit coupling in this study. Surface dangling bonds were passivated by giving an excess energy of $30 \mathrm{eV}$ according to the $s p^{3}$ hybridization scheme. ${ }^{21}$ The tight binding method gives relatively accurate results and is suitable for nanostructures, so this method is often used to calculate the band structure of nanowires. However, this method takes long calculation time and it is difficult to relate the calculation results by TB to bulk $E-k$ dispersion. 

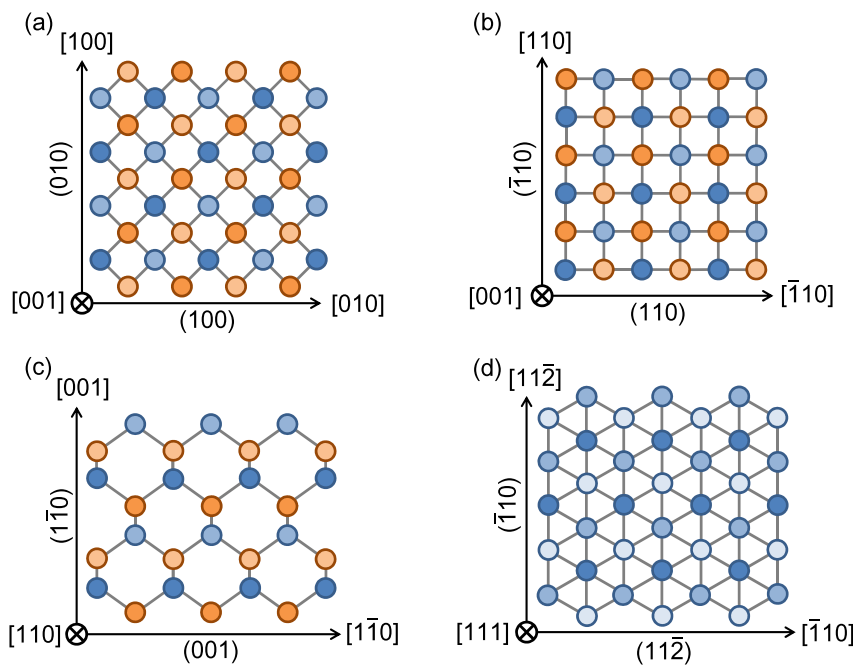

FIG. 1. Crystal structures of targeted GaAs nanowires. Each figure illustrates (a) $[001] /(010) /(100)$, (b) $[001] /(\overline{1} 10) /(110)$, (c) $[110] /(1 \overline{1} 0) /(001)$, and (d) $[111] /(\overline{1} 10) /(11 \overline{2})$ nanowires. The blue circles represent $\mathrm{Ga}$ atoms, and the orange ones represent As atoms.

In DEME, the effective mass equation is discretized and numerically solved. The effective mass equation for an envelope function $F(\boldsymbol{r})$ is written as ${ }^{22}$

$$
\left[E_{0}(-i \nabla)+H_{1}(\boldsymbol{r})\right] F(\boldsymbol{r})=E F(\boldsymbol{r}),
$$

where $E_{0}(\boldsymbol{k})$ is the bulk $E-k$ dispersion and $H_{1}(\boldsymbol{r})$ is the perturbation potential. Here, $E_{0}(\boldsymbol{k})$ is calculated by TB for the conduction band in a periodic zone scheme. To discretize the effective mass equation in a cross-sectional plane (a plane perpendicular to the nanowire orientation), $N$ lattice points were assumed in a supercell with an area of $S$. Here, $x$ and $y$ axes were defined inside the cross-sectional plane and $z$-axis was along the nanowire orientation. The wave function was expanded by a basis localized at each lattice point $\left(x_{j}, y_{j}\right)$, which was made by superposing plane waves. Therefore, this calculation is equivalent to that by a plane wave basis set. Numerical calculation of the effective mass equation using a plane wave basis set has been already reported. ${ }^{23}$ The conduction band valleys should be carefully taken into account to obtain accurate band structure. We properly arranged the lattice points for each nanowire orientation and substrate face in the present work. Details about this computational meshing are described in Appendix B. The perturbation potential $H_{1}(\boldsymbol{r})$ for each basis function $a\left(x-x_{j}, y-y_{j}\right)$ is approximated by the value at the lattice point $\left(x_{j}, y_{j}\right)$. The discrete effective mass equation is given by (Appendix A)

$$
\begin{aligned}
& \sum_{j=1}^{N}\left[\frac{1}{N} \sum_{k_{x}, k_{y}} e^{i k_{x}\left(x_{m}-x_{j}\right)+i k_{y}\left(y_{m}-y_{j}\right)} E_{0}\left(k_{x}, k_{y}, k_{z}\right)+H_{1}\left(x_{j}, y_{j}\right) \delta_{m, j}\right] \\
& \quad \times F\left(x_{j}, y_{j}\right)=E\left(k_{z}\right) F\left(x_{m}, y_{m}\right),
\end{aligned}
$$

and solving Eq. (2) for each wavenumber $k_{z}$ leads to the $E-k$ dispersion of nanowires $E\left(k_{z}\right)$. The confinement potential of $30 \mathrm{eV}$ was applied as $H_{1}\left(x_{j}, y_{j}\right)$ in the barrier layer.

DEME is less computationally demanding than TB, though it is less accurate. Furthermore, by DEME, it is easy to analyze quantum confinement effects in terms of bulk $E-k$ dispersion, because DEME directly uses bulk $E$ - $k$ dispersion.

\section{B. Nanowire structures}

Targeted GaAs nanowires have zinc-blende structure and rectangular cross-sections with a width and height of 2-10 nm. Their axial orientation and two side faces are $[001] /(010) /(100), \quad[001] /(\overline{1} 10) /(110), \quad[110] /(1 \overline{1} 0) /(001)$, and $[111] /(\overline{1} 10) /(11 \overline{2})$. These crystal structures are shown in Fig. 1. Since we focus on the understanding of quantum-confinement effects on electrons in this study, we neglected the effect of strain, relaxation, and surface reconstruction. One side of crosssections was kept at $10 \mathrm{~nm}$ (height) and the other side (width) was varied from $2 \mathrm{~nm}$ to $10 \mathrm{~nm}$ as shown in Fig. 2.

\section{CALCULATION RESULTS}

Figure 3 shows the conduction band structure of [110] GaAs nanowires with various cross-sectional shapes calculated by TB and DEME. In the figure, $a_{0}$ means the lattice constant of bulk GaAs, 0.5653 nm. ${ }^{24}$ Figures $3(\mathrm{a})-3(\mathrm{c})$ are the conduction band structures of $10 \mathrm{~nm} \times 2 \mathrm{~nm}, 2 \mathrm{~nm}$ $\times 10 \mathrm{~nm}$, and $10 \mathrm{~nm} \times 10 \mathrm{~nm}$ cross-sectional nanowires, respectively, which were calculated by TB. The TB calculation revealed that, for all the structures considered in this study, the lowest conduction subband is located at $k=0$ ( $\Gamma$ valley) and the energy difference between the lowest conduction subband and the second lowest is larger than $70 \mathrm{meV}$. Therefore, the effective mass of the lowest subband of the $\Gamma$ valley is important to predict the electron transport characteristics. This lowest subband of the $\Gamma$ valley naturally originates from the $\Gamma$ valley of bulk GaAs. Figure 3(d) shows the conduction band structure of a $10 \mathrm{~nm} \times 10 \mathrm{~nm}$ cross-sectional nanowire calculated by DEME. Comparing Figs. 3(c) and 3(d), for relatively large cross-sectional sizes such as $10 \mathrm{~nm} \times 10 \mathrm{~nm}$, DEME gives similar band structure to TB.
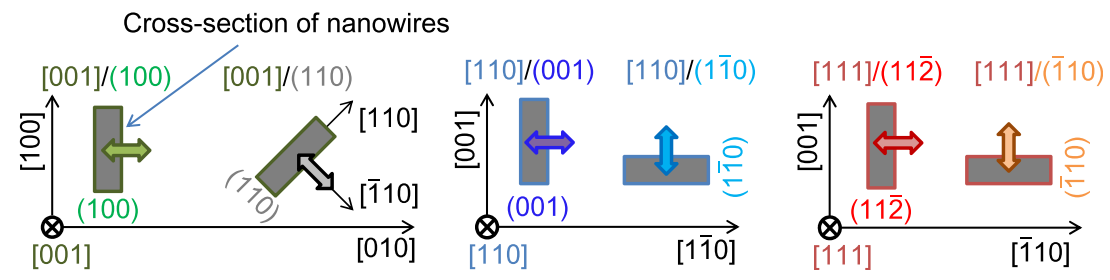

FIG. 2. Cross-sectional shapes of targeted rectangular GaAs nanowires. One side (height) is kept at $10 \mathrm{~nm}$ and the other (width) is varied from $2 \mathrm{~nm}$ to $10 \mathrm{~nm}$. The orientations of nanowires targeted in this study are [001], [110], and [111], and for each wire orientation, two types of substrate faces (perpendicular to the "height" direction) are considered. 
(a) TB

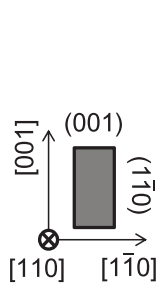

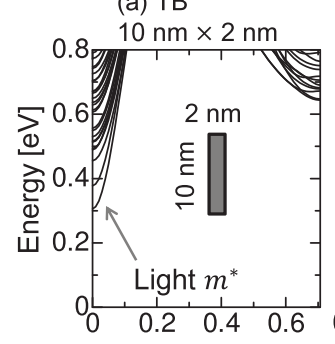

(b) TB

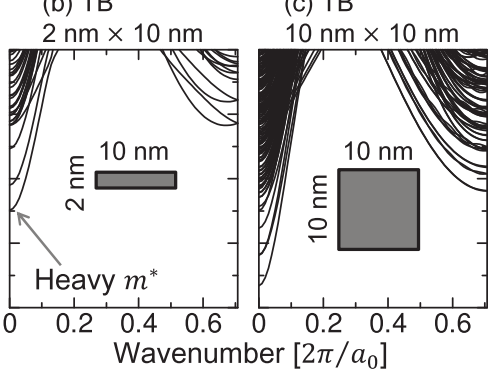

(d) DEME

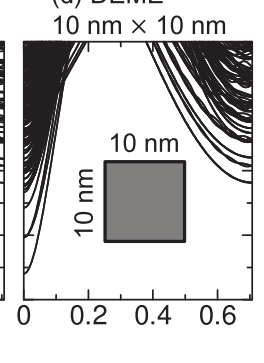

FIG. 3. Conduction band structure of [110] GaAs nanowires. (a) $10 \mathrm{~nm} \times 2 \mathrm{~nm}$, calculated by TB. (b) $2 \mathrm{~nm} \times 10 \mathrm{~nm}$, calculated by TB. (c) $10 \mathrm{~nm} \times 10 \mathrm{~nm}$, calculated by TB. (d) $10 \mathrm{~nm} \times 10 \mathrm{~nm}$, calculated by DEME. The energy is referenced from the conduction band edge of bulk GaAs. $\Gamma$ valley at $k=0$ originates mainly from the bulk $\Gamma$ valley and off- $\Gamma$ valley at the zone edge with higher energy originates mainly from bulk $\mathrm{L}$ valleys. The conduction band structure by DEME shows good agreement to that by TB when the width and height are larger than $5 \mathrm{~nm}$.

Figure 4 shows the width dependence of quantum-confinement-induced energy shift of the $\Gamma$ valley calculated by TB and DEME. The height of nanowires was kept at $10 \mathrm{~nm}$ and the width was varied. The energy shift by quantum confinement is almost independent of the wire orientations as far as the wire width is the same. This implies that the anisotropy of the $\Gamma$ valley of bulk GaAs gives small effects on the energy shift at $\Gamma$ point in nanowires.

Figure 5 shows the width dependence of electron effective mass at the $\Gamma$ valley. The effective mass is strongly dependent on the wire orientations at each wire size. In [001] nanowires, effective mass significantly increases as the cross-sectional size decreases. On the contrary, in [111] nanowires, the increase of effective mass caused by quantum confinement is smaller than [001] nanowires. In [110] nanowires, the width dependence of effective mass shows strong anisotropy in the confinement direction. The effective mass of [110] nanowires increases as the width along [001] decreases ([110]/(110) nanowires). It is, however, insensitive to the size variation along [1] 0$]([110] /(001)$ nanowires).

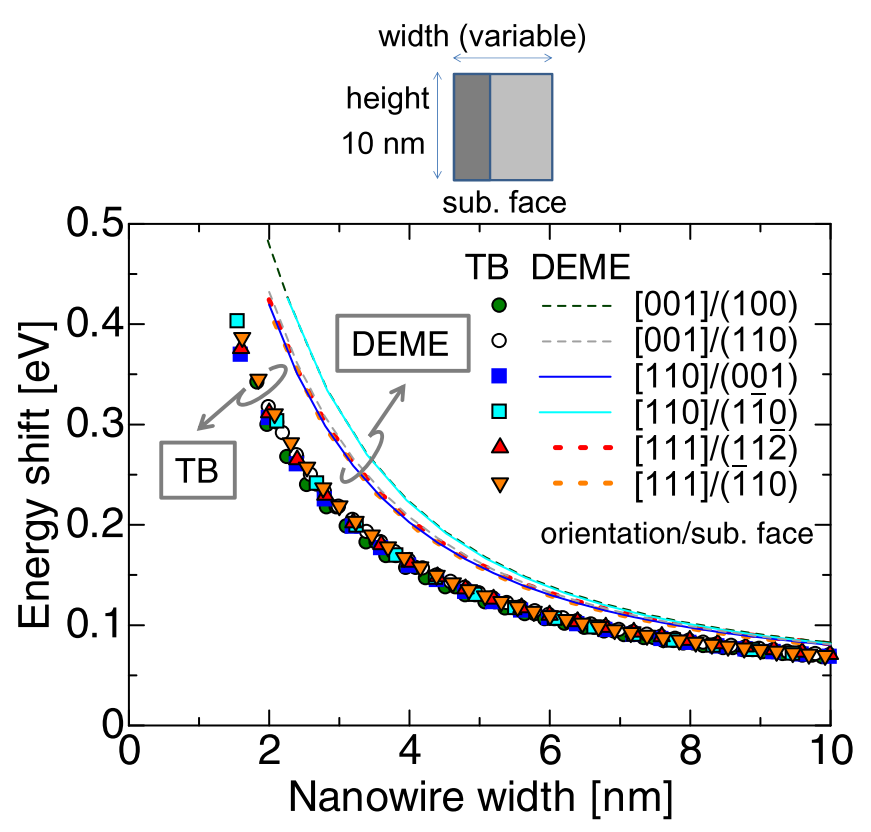

FIG. 4. Width dependence of the energy shift of $\Gamma$ valley by quantum confinement in rectangular cross-sectional GaAs nanowires with different orientations and substrate faces. The width along the substrate face was varied while the height was kept at $10 \mathrm{~nm}$.
The effective mass calculated by DEME shows a similar tendency to that calculated by TB, and also good quantitative agreement with that for nanowires with a cross-sectional size larger than $5 \mathrm{~nm}$. This indicates that the size dependence of effective mass can be explained in terms of the $E-k$ dispersion of bulk GaAs because DEME results reflect the band structure of bulk GaAs. The increase of effective mass by quantum confinement is due to the nonparabolicity of the $\Gamma$ valley of bulk GaAs, and the dependence of effective mass on the wire orientations and substrate faces is caused by the anisotropy of the bulk $\Gamma$ valley.

\section{DISCUSSION}

The size dependence of electron effective mass calculated by TB and DEME depends on the wire orientations and substrate faces, and the trend is the same for TB and DEME. Therefore, this dependence can be explained by the nonparabolicity and anisotropy of bulk $\Gamma$ valley. In this section, we discuss the dependence of effective mass on wire geometry,

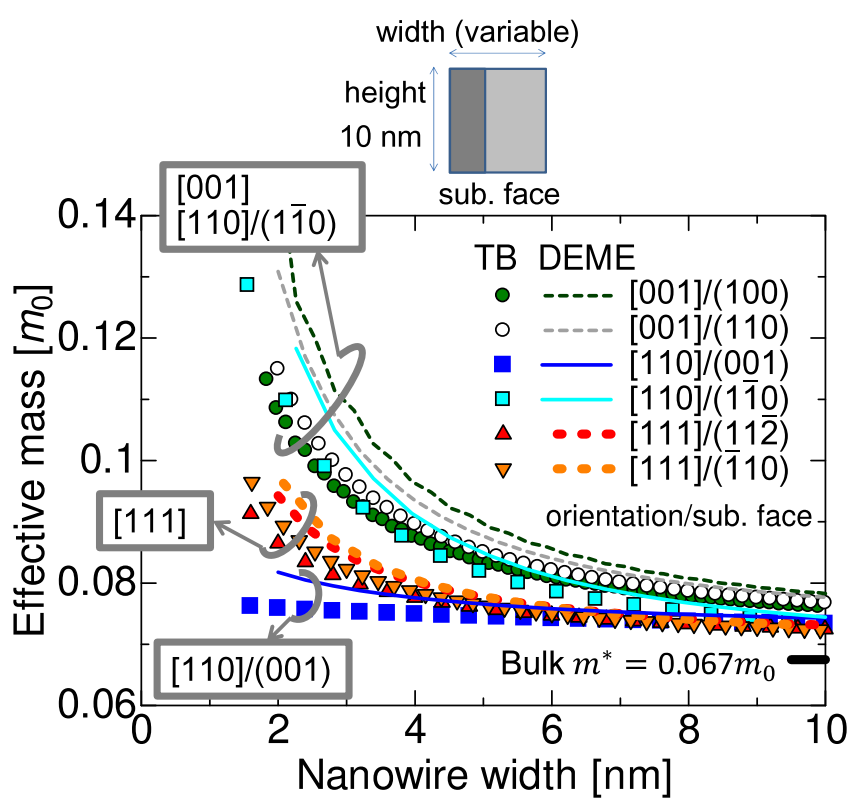

FIG. 5. Width dependence of electron effective mass in rectangular crosssectional GaAs nanowires with different orientations and substrate faces. The height was kept at $10 \mathrm{~nm}$ and the width was varied. 
(a) Probability density in real-space

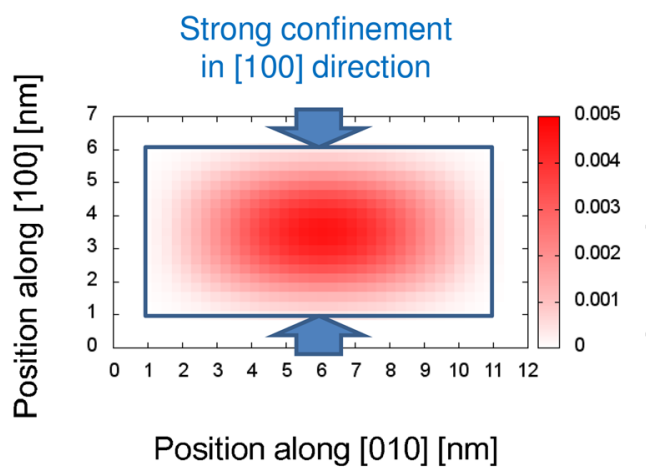

(b) Probability density in k-space

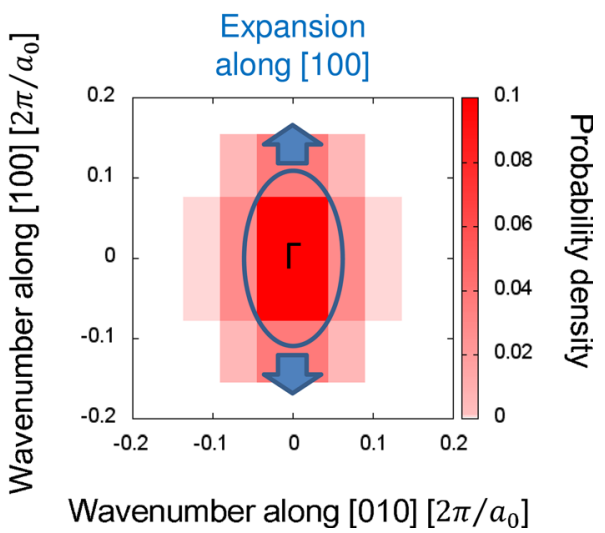

FIG. 6. Probability density of a [001]-oriented GaAs nanowire with cross-sectional size of $5 \mathrm{~nm} \times 10 \mathrm{~nm}$ calculated by DEME. When the wave function is strongly confined in [100] direction in real-space (a), the wavefunction in $k$-space (b) expands along [100] direction.

such as cross-sectional shapes and orientations, in terms of the $E$ - $k$ dispersion of $\Gamma$ valley of bulk GaAs.

To understand how the effective mass of nanowires depends on wire geometry, first, we discuss the $E-k$ dispersion of nanowires $E\left(k_{z}\right)$ at the $\Gamma$ valley. When the wavenumber along the nanowire $k_{z}$ increases from $k_{z}=0$, the larger the energy increase is, the lighter the effective mass is. Therefore, it is essential to discuss the energy increase in nanowires as the wavenumber increases, based on bulk $E-k$ dispersion $E_{0}\left(k_{x}, k_{y}, k_{z}\right)$, and DEME is suitable for this purpose. Here, a $k$-space plane vertical to the wire orientation is considered. This plane is represented by $k_{z}=$ constant. In DEME, bulk $E-k$ dispersion in each $k$-space plane is used to calculate the quantum confinement effects. The calculated real-space wave function by DEME can be transformed to $k$-space wave function $F_{K}\left(k_{x}, k_{y}\right)$. From the $k$-space probability density $\left|F_{K}\left(k_{x}, k_{y}\right)\right|^{2}$, we can see what $k$-components contribute to the wave function (Fig. 6). Next, we calculated the energy increase in 3-D $E-k$ dispersion with the wavenumber along the nanowire, in other words, the energy difference between two $k$-space planes, $k_{z}$ $=0$ and $k_{z}=\delta$, where $\delta$ is a small positive value. This energy difference $E_{0}\left(k_{x}, k_{y}, \delta\right)-E_{0}\left(k_{x}, k_{y}, 0\right)$ reflects how the bulk energy increases with the wavenumber along the nanowire $k_{z}$. By combining this with the $k$-space probability density, the energy increase in nanowires with $k_{z}, E(\delta)-E(0)$, and thereby the effective mass in nanowires, can be estimated.

More rigorously, this can be described as a first order perturbation to the DEME in $k$-space. The variation of bulk $E-k$ dispersion $E_{0}\left(k_{x}, k_{y}, \delta\right)-E_{0}\left(k_{x}, k_{y}, 0\right)$ is regarded as a perturbation "potential" in $k$-space, and then the energy variation by first-order perturbation is given by

$$
\sum_{k_{x}, k_{y}}\left|F_{K}\left(k_{x}, k_{y}\right)\right|^{2}\left\{E_{0}\left(k_{x}, k_{y}, \delta\right)-E_{0}\left(k_{x}, k_{y}, 0\right)\right\} .
$$

This means the average of the energy increase $E_{0}\left(k_{x}, k_{y}, \delta\right)$ $-E_{0}\left(k_{x}, k_{y}, 0\right)$ weighted by the probability density in $k$-space $\left|F_{K}\left(k_{x}, k_{y}\right)\right|^{2}$.

Based on the discussion above, we explain how the effective mass of GaAs nanowires depends on crosssectional shapes. In Fig. 7, the difference of energy between two $k$-space planes, $k_{z}=0$ and $k_{z}=\delta=0.01 \times 2 \pi / a_{0}$, is plotted against the wavenumbers in these $k$-planes.

First, we consider [001] GaAs nanowires (Fig. 7(a)). When the cross-section of a nanowire is large in real space, the $k$-components near the bulk $\Gamma$ point, or with small $k$, contribute to the wave function in $k$-space. Since the energy increase is large for these $k$-components, thick nanowires have light effective mass. When the wave function is strongly confined in the [100] direction in real-space, however, the wave function in $k$-space expands along the [100] direction as shown in Fig. 6. Because this wave function includes $k$-components with small energy increase with $k_{z}$, the increase in the electron energy in the nanowire, $E(\delta)-E(0)$, becomes small and the effective mass becomes heavy. This is why the effective mass of [001] nanowires becomes heavy when the crosssectional size is small. In a similar manner, as shown in Fig. 7(b), strong confinement in the [1 10$]$ direction makes the effective mass of [001] nanowires heavy.

Next the case of [110] nanowires with strong confinement in the [1 10 ] direction (Fig. 7(c)) is considered. In this case, the wave function is strongly confined in the [110] direction in real-space, thus, the wave function expands along the [110] direction in $k$-space. However, this state is made of $k$-components with large energy increase, and the effective mass stays light (Fig. 3(a)). In the case of [110] nanowires with strong confinement in the [001] direction, $k$-space wave function expands along the [001] direction and $k$-components with small energy increase contribute to the wave function. Therefore, the energy increase with wavenumber along the nanowire becomes small, and the effective mass becomes heavy by width reduction along the [001] direction (Fig. 3(b)).

Finally, in [111] nanowires (Fig. 7(d)), as the crosssectional size decreases, $k$-components with energy decrease and with large energy increase contribute simultaneously. Since this situation is common to both $[111] /(\overline{1} 10)$ and $[111] /(11 \overline{2})$ nanowires, these nanowires experience small increase in effective mass. The method used in this discussion will be able to be applied to other III-V semiconductor nanowires. Similar discussion may be possible directly for tight-binding wave functions by projecting them on bulk Bloch functions. 
(a)

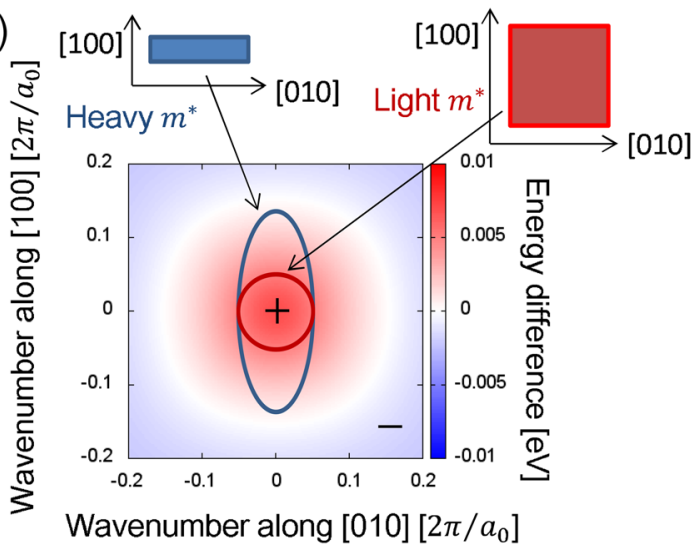

(c)

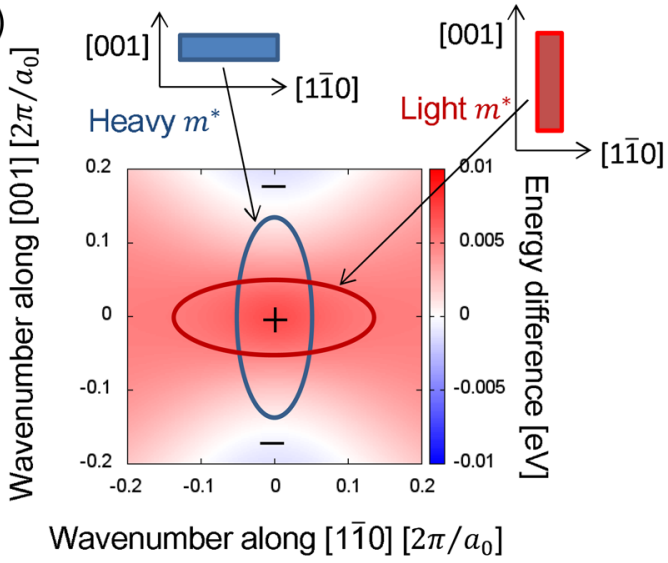

(b)

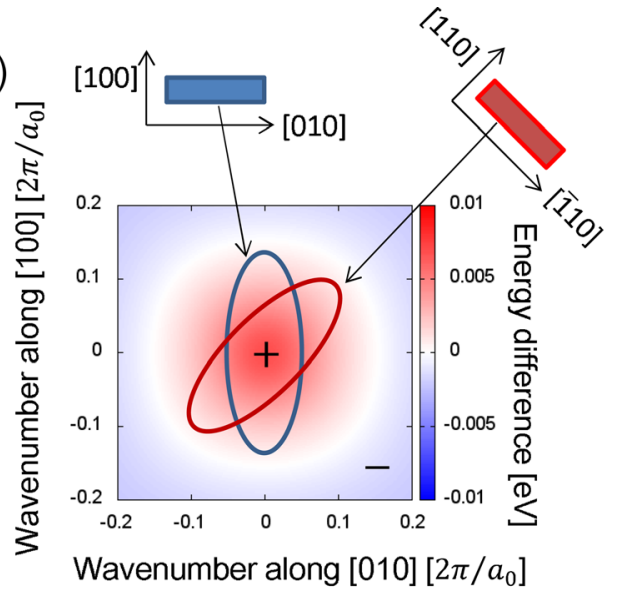

(d)

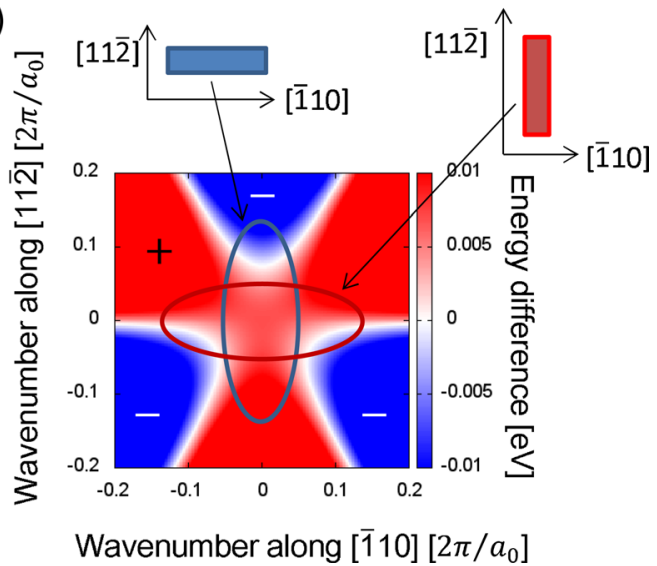

FIG. 7. Understanding of the dependence of electron effective mass in GaAs nanowires on wire geometry. Energy difference $E_{0}\left(k_{x}, k_{y}, 0.01 \times 2 \pi / a_{0}\right)-$ $E_{0}\left(k_{x}, k_{y}, 0\right)$ is plotted. The signs "+" and "-" in the figures show the sign of energy difference. The smaller the energy difference is, the heavier the effective mass becomes. (a) In [001] nanowires, the effective mass increases as the cross-sectional size decreases. (b) The increase in effective mass of [001] nanowires is almost independent of substrate faces. (c) In [110] nanowires, the effective mass increases as the width along [001] decreases, however, it is insensitive to the size variation along [1ㅣㅣ. (d) In [111] nanowires, the increase of effective mass caused by size decrease is small.

\section{CONCLUSION}

We calculated the conduction band structure of rectangular cross-sectional GaAs nanowires with various orientations and substrate faces using a tight-binding method and a discrete effective mass equation, which is based on bulk $E-k$ dispersion. Taking account of the electron effective mass of the lowest conduction subband, [110]-oriented nanowires with small width along [001] and thin [001]-oriented nanowires are unfavorable for $n$-channel material because they have heavy effective mass. In contrast, [110]-oriented nanowires with wider width along [001] than along [11 0$]$ are favorable for $\mathrm{n}$ channel material because they have light effective mass even when the cross-sectional size is small. This trend can be explained by the anisotropy of $\Gamma$ valley of bulk GaAs.

\section{APPENDIX A: DERIVATION OF DISCRETE EFFECTIVE MASS EQUATION}

Here, we derive the DEME (Eq. (2)). First, $F(\boldsymbol{r})$ in Eq. (1) is expanded using a basis localized at each lattice point $\left(x_{j}, y_{j}\right)$

$$
a\left(x-x_{j}, y-y_{j}\right)=\frac{1}{\sqrt{N}} \sum_{k_{x}, k_{y}} e^{-i k_{x} x_{j}-i k_{y} y_{j}} \frac{1}{\sqrt{S}} e^{i k_{x} x+i k_{y} y} .
$$

This corresponds to the Fourier expansion representation of a two-dimensional Dirac's delta function in the continuous limit. The result is

$$
F(\boldsymbol{r})=\sum_{j=1}^{N} \frac{1}{\sqrt{N}} F\left(x_{j}, y_{j}\right) a\left(x-x_{j}, y-y_{j}\right) \times \exp \left(i k_{z} z\right),
$$

where $z$ is along the nanowire axis and $j$ denotes $N$ $=N_{x} \times N_{y}$ lattice points in the cross-sectional plane with an area of $L_{x} \times L_{y}=S$. In Eq. (A1), the wavenumbers $k_{x}, k_{y}$ are limited by the two-dimensional periodic boundary condition

$$
\begin{aligned}
& k_{x}=\frac{2 \pi}{L_{x}}\left(-\frac{N_{x}}{2}+1\right), \frac{2 \pi}{L_{x}}\left(-\frac{N_{x}}{2}+2\right), \ldots, \frac{2 \pi}{L_{x}}\left(\frac{N_{x}}{2}\right), \\
& k_{y}=\frac{2 \pi}{L_{y}}\left(-\frac{N_{y}}{2}+1\right), \frac{2 \pi}{L_{y}}\left(-\frac{N_{y}}{2}+2\right), \ldots, \frac{2 \pi}{L_{y}}\left(\frac{N_{y}}{2}\right),
\end{aligned}
$$

where $N_{x}$ and $N_{y}$ are even numbers. The basis shown in Eq. (A1) is orthonormal

$$
\int_{S} a^{*}\left(x-x_{m}, y-y_{m}\right) a\left(x-x_{j}, y-y_{j}\right) \mathrm{d} x \mathrm{~d} y=\delta_{m, j} .
$$


(a) $[001] /(100)$

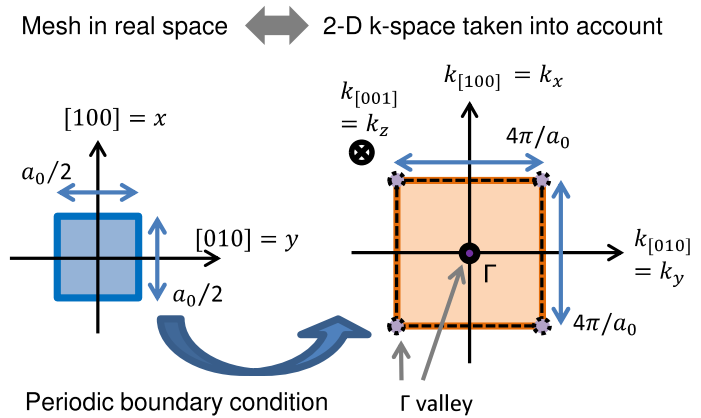

(c) [110]

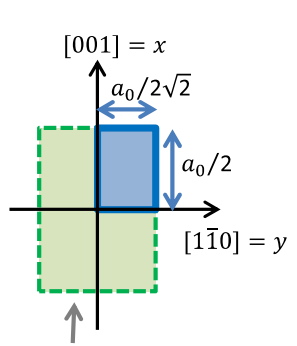

4 cells are treated as one large cell.

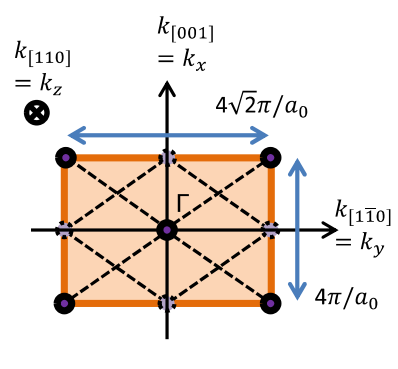

$4 \pi / a_{0}$ (b) $[001] /(110)$

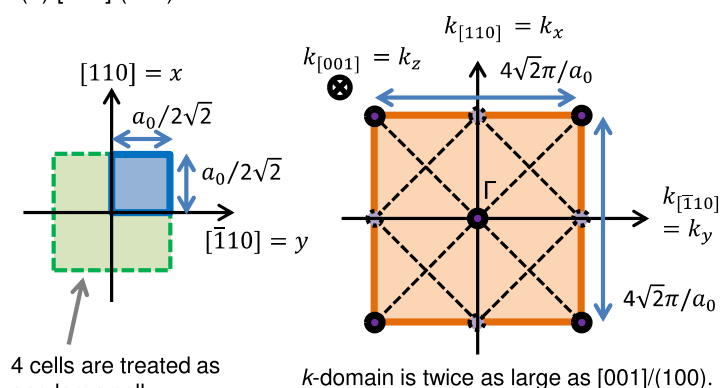

one large cell. $k$-domain is twice as large as [001]/(100). (d) [111]

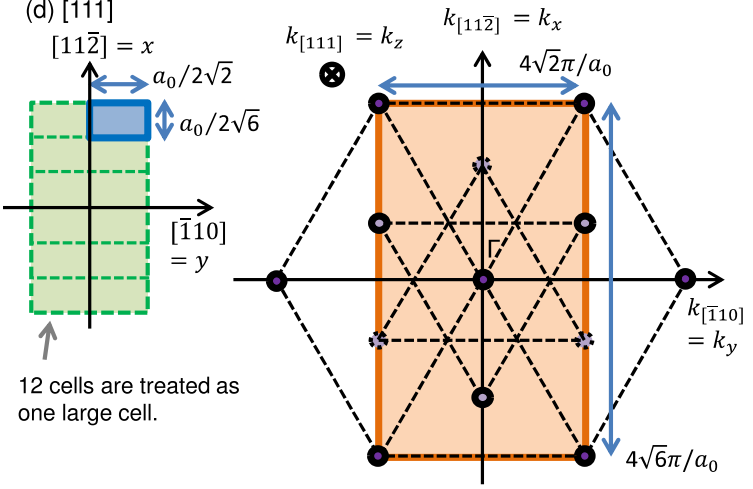

FIG. 8. Computational mesh and the area of $k$-space considered for each wire geometry. $z$-axis is defined along the nanowire orientation and $x$ and $y$-axes are in the cross-sectional plane. $\Gamma$ valleys in periodic zone scheme are shown by circles, and those at different values of $k_{z}$ are shown by different symbols. The size of real-space mesh is related to the range of considered 2-D $k$-space via the periodic boundary condition (Eq. (A3)). (a) [001]/(100) nanowires. (b) $[001] /(110)$ nanowires. (c) [110] nanowires. (d) [111] nanowires.

Substituting Eq. (A2) into the effective mass equation Eq. (1), we get

$$
\begin{aligned}
0 & =\left[E_{0}(-i \nabla)+H_{1}(\boldsymbol{r})-E\right] \sum_{j=1}^{N} \frac{1}{\sqrt{N}} F\left(x_{j}, y_{j}\right) a\left(x-x_{j}, y-y_{j}\right) \times \exp \left(i k_{z} z\right) \\
& \simeq \sum_{j=1}^{N} \frac{1}{\sqrt{N}} F\left(x_{j}, y_{j}\right) \sum_{k_{x}, k_{y}} \frac{1}{\sqrt{N}} e^{-i k_{x} x_{j}-i k_{y} y_{j}} E_{0}\left(k_{x}, k_{y}, k_{z}\right) \frac{1}{\sqrt{S}} e^{i k_{x} x+i k_{y} y} \times \exp \left(i k_{z} z\right) \\
& +\sum_{j=1}^{N} \frac{1}{\sqrt{N}} F\left(x_{j}, y_{j}\right)\left[H_{1}\left(x_{j}, y_{j}\right)-E\right] a\left(x-x_{j}, y-y_{j}\right) \times \exp \left(i k_{z} z\right) .
\end{aligned}
$$

Here, we replaced $H_{1}(\boldsymbol{r}) a\left(x-x_{j}, y-y_{j}\right)$ by $H_{1}\left(x_{j}, y_{j}\right) a\left(x-x_{j}, y-y_{j}\right)$ because $a\left(x-x_{j}, y-y_{j}\right)$ is localized and $H_{1}$ is independent of $z$ as it is the confinement potential of the nanowire. Multiplying this by $a^{*}\left(x-x_{m}, y-y_{m}\right)$ and integrating using the orthonormality of $a\left(x-x_{j}, y-y_{j}\right)$ and plane waves $\frac{1}{\sqrt{S}} e^{i k_{x} x+i k_{y} y}$, finally we get Eq. (2) (DEME)

$$
\begin{aligned}
0 \simeq & \sum_{j=1}^{N} F\left(x_{j}, y_{j}\right) \sum_{k_{x}, k_{y}} \frac{1}{\sqrt{N}} e^{-i k_{x} x_{j}-i k_{y} y_{j}} E_{0}\left(k_{x}, k_{y}, k_{z}\right) \int_{S} a^{*}\left(x-x_{m}, y-y_{m}\right) \frac{1}{\sqrt{S}} e^{i k_{x} x+i k_{y} y} \mathrm{~d} x \mathrm{~d} y \\
& +\sum_{j=1}^{N} F\left(x_{j}, y_{j}\right)\left[H_{1}\left(x_{j}, y_{j}\right)-E\right] \int_{S} a^{*}\left(x-x_{m}, y-y_{m}\right) a\left(x-x_{j}, y-y_{j}\right) \mathrm{d} x \mathrm{~d} y \\
= & \sum_{j=1}^{N} F\left(x_{j}, y_{j}\right) \sum_{k_{x}, k_{y}} \frac{1}{\sqrt{N}} e^{-i k_{x} x_{j}-i k_{y} y_{j}} E_{0}\left(k_{x}, k_{y}, k_{z}\right) \frac{1}{\sqrt{N}} e^{i k_{x} x_{m}+i k_{y} y_{m}}+\sum_{j=1}^{N} F\left(x_{j}, y_{j}\right)\left[H_{1}\left(x_{j}, y_{j}\right)-E\right] \delta_{m, j} \\
= & \sum_{j=1}^{N}\left[\frac{1}{N} \sum_{k_{x}, k_{y}} e^{i k_{x}\left(x_{m}-x_{j}\right)+i k_{y}\left(y_{m}-y_{j}\right)} E_{0}\left(k_{x}, k_{y}, k_{z}\right)+H_{1}\left(x_{j}, y_{j}\right) \delta_{m, j}\right] F\left(x_{j}, y_{j}\right)-E F\left(x_{m}, y_{m}\right) .
\end{aligned}
$$




\section{APPENDIX B: COMPUTATIONAL MESH FOR DISCRETE EFFECTIVE MASS EQUATION}

In this section, we explain the computational meshes of DEME for different wire geometries. The meshes in real space are determined to cover the rectangular unit cell in two-dimensional $k$-space based on the periodic boundary condition (Eq. (A3)). Since the geometry of the rectangular unit cell in two-dimensional $k$-space is dependent on wire orientations and substrate faces, the computational mesh in real space is also dependent on wire orientations and substrate faces.

In the case of $[001] /(100)$ nanowires, the unit cell in the two-dimensional $k$-space is $-2 \pi / a_{0}<k_{x} \leq 2 \pi / a_{0}$, $-2 \pi / a_{0}<k_{y} \leq 2 \pi / a_{0}$ as shown in Fig. 8(a). To consider this range, we chose the computational mesh of a square with the size of $a_{0} / 2 \times a_{0} / 2$ in the real space. According to the periodic boundary condition (Eq. (A3)), the range of the considered 2-D $k$-space agrees with the unit cell in the 2-D $k$-space.

In $[001] /(110)$ nanowires, the unit cell in the 2-D $k$ space is the same as $[001] /(100)$ nanowires. However, when we deal with rectangular cross-sectional nanowires, it is more convenient to take the mesh along the [110] and [1 10] directions than along the [100] and [010] directions. Therefore, we adopted the mesh with the size of $a_{0} / 2 \sqrt{2}$ $\times a_{0} / 2 \sqrt{2}$ as shown in Fig. 8(b). In this case, the area of the 2-D $k$-space considered is twice as large as that of [001]/(100) nanowires. At $k_{z}=0$, two $\Gamma$ valleys at $\left(k_{x}, k_{y}\right)$ $=(0,0),\left(2 \sqrt{2} \pi / a_{0}, 2 \sqrt{2} \pi / a_{0}\right)$ are considered. This leads to twice as many eigen states in calculated results. To eliminate these excess states, 4 cells are treated as one large cell as in Fig. 8(b) and the same $H_{1}$ was applied to these 4 cells. The difference between the two equivalent states originating from the two $\Gamma$ valleys at $\left(k_{x}, k_{y}\right)=(0,0),\left(2 \sqrt{2} \pi / a_{0}\right.$, $\left.2 \sqrt{2} \pi / a_{0}\right)$ is only the vibration inside this large cell, and these states are two-fold degenerate. By adopting only one state of each two-fold degenerate pair, we can get a proper set of eigen values. Also, valleys at $k_{z}=0$ and at $k_{z}=2 \pi / a_{0}$ give equivalent eigen states with the same energy and different vibration inside the large cell. This realizes the proper periodicity about the $E-k$ dispersion of a [001] nanowire.
We can use a similar method to choose the mesh for [110] nanowires as shown in Fig. 8(c). The procedure used for $[001] /(110)$ nanowires, adopting only one state of each two-fold degenerate pair, is common to [110] and [111] nanowires.

In the case of [111] nanowires, we can use a large cell with the size of $3 a_{0} / \sqrt{6} \times a_{0} / \sqrt{2}$ consisting of $6 \times 2$ cells with the size of $a_{0} / 2 \sqrt{6} \times a_{0} / 2 \sqrt{2}$. This allows all the equivalent $\Gamma$ valleys at different $k_{z}$ in Fig. 8(d) to give equivalent states except for the vibration inside the large cell.

${ }^{1}$ J.-T. Park and J.-P. Colinge, IEEE Trans. Electron Devices 49, 2222 (2002).

${ }^{2}$ Y. Cui, Z. Zhong, D. Wang, W. U. Wang, and C. M. Lieber, Nano Lett. 3, 149 (2003).

${ }^{3}$ J. Goldberger, A. I. Hochbaum, R. Fan, and P. Yang, Nano Lett. 6, 973 (2006).

${ }^{4}$ N. Singh, A. Agarwal, L. Bera, T. Liow, R. Yang, S. Rustagi, C. Tung, R. Kumar, G. Lo, N. Balasubramanian, and D. Kwong, IEEE Electron Device Lett. 27, 383 (2006).

${ }^{5}$ Y. Xuan, Y. Q. Wu, and P. D. Ye, IEEE Electron Device Lett. 29, 294 (2008).

${ }^{6}$ J. A. del Alamo, Nature 479, 317 (2011).

${ }^{7}$ A. W. Dey, C. Thelander, E. Lind, K. A. Dick, B. M. Borg, M. Borgstrom, P. Nilsson, and L.-E. Wernersson, IEEE Electron Device Lett. 33, 791 (2012).

${ }^{8}$ J. J. Hou, F. Wang, N. Han, H. Zhu, K. Fok, W. Lam, S. Yip, T. Hung, J. E.-Y. Lee, and J. C. Ho, Appl. Phys. Lett. 102, 093112 (2013).

${ }^{9}$ Y. Arakawa, T. Yamauchi, and J. N. Schulman, Phys. Rev. B 43, 4732 (1991).

${ }^{10}$ M. P. Persson and H. Q. Xu, Appl. Phys. Lett. 81, 1309 (2002).

${ }^{11}$ M. P. Persson and H. Q. Xu, Nano Lett. 4, 2409 (2004).

${ }^{12}$ M. Luisier, A. Schenk, W. Fichtner, and G. Klimeck, Phys. Rev. B 74, 205323 (2006).

${ }^{13}$ A. Franceschetti and A. Zunger, J. Chem. Phys. 104, 5572 (1996).

${ }^{14}$ J. Xia and K. W. Cheah, Phys. Rev. B 55, 15688 (1997).

${ }^{15}$ S. Cahangirov and S. Ciraci, Phys. Rev. B 79, 165118 (2009).

${ }^{16}$ M. Rosini and R. Magri, ACS Nano 4, 6021 (2010).

${ }^{17}$ P. Lu, H. Cao, X. Zhang, Z. Yu, N. Cai, T. Gao, and S. Wang, Physica E 52, 34 (2013).

${ }^{18}$ J. M. Jancu, R. Scholz, F. Beltram, and F. Bassani, Phys. Rev. B 57, 6493 (1998).

${ }^{19}$ J. C. Slater and G. F. Koster, Phys. Rev. 94, 1498 (1954).

${ }^{20}$ N. Morioka, H. Yoshioka, J. Suda, and T. Kimoto, J. Appl. Phys. 109, 064318 (2011).

${ }^{21}$ S. Lee, F. Oyafuso, P. Allmen, and G. Klimeck, Phys. Rev. B 69, 045316 (2004).

${ }^{22} \mathrm{P}$. Y. Yu and M. Cardona, Fundamentals of Semiconductors, 4th ed. (Springer, Berlin, 2010).

${ }^{23}$ A. Mirčetić, Z. Ikonić, V. Milanović, and D. Indjin, J. Appl. Phys. 92, 515 (2002).

${ }^{24}$ S. M. Sze and K. K. Ng, Physics of Semiconductor Devices, 3rd ed. (Wiley, New York, 2006). 